\title{
Imaging T Cells In Vivo
}

\author{
Dario Neri
}

Department of Chemistry and Applied Biosciences, Swiss Federal Institute of Technology (ETH Zürich), Zürich, Switzerland

$\mathbf{T}$ here is a growing pharmaceutical and biomedical interest in the opportunity to boost or inhibit the activity of the immune system at the site of disease. The experimental demonstration that most human tumors contain at least 1 mutation per megabase (i.e., more than 1,000 mutations in the whole genome (1)) suggests that various mutated peptides may be presented on major histocompatibility complex class I molecules (human leukocyte antigen class $\mathrm{I}$ in humans) and be recognized by specific $\mathrm{T}$ cells, thus serving as potential tumor rejection antigens. The existence of such peptides provides a strong rationale for the design and implementation of immunotherapeutic strategies against various types of malignancies. More broadly, T cell-based recognition of peptides presented on major histocompatibility complex class I and class II molecules is crucially important for a broad variety of disease conditions, including chronic inflammatory processes, organ rejection, autoimmunity, and allergy.

\section{See page 1258}

Antibodies that target the inhibitory proteins CTLA-4 and PD-1 act as potent stimulators of the immune system and have gained marketing authorization (or are in advanced clinical trials) for the treatment of various types of cancer, including metastatic melanoma (2-4). Moreover, antibody-cytokine fusion proteins, capable of selective localization in cancer or at sites of chronic inflammation, are currently being investigated in clinical trials, with the potential to selectively boost or inhibit immunity at the site of disease (5-9). These and other immunomodulatory strategies crucially affect the activity of $\mathrm{T}$ cells. In addition, the genetic engineering of artificial receptors on $\mathrm{T}$ cells is opening new avenues for the treatment of hematologic malignancies and, potentially, other tumor types (10).

The opportunity to study $\mathrm{T}$ cell activities in humans is often limited and, in most cases, restricted to the ex vivo analysis of lymphocytes in biopsies (e.g., staining of lymphocyte infiltrates) or in blood (e.g., by the tetramer-assisted investigation of $\mathrm{T}$ cell specificities by fluorescence-activated cell sorter $(11,12))$. The ability to image $\mathrm{T}$ cells in vivo could open novel biomedical

Received May 8, 2015; revision accepted May 11, 2015.

For correspondence or reprints contact: Dario Neri, Department of Chemistry and Applied Biosciences, Swiss Federal Institute of Technology (ETH Zürich), Vladimir-Prelog-Weg 4, CH-8093 Zürich, Switzerland.

E-mail: neri@pharma.ethz.ch

Published online May 21, 2015.

COPYRIGHT (C) 2015 by the Society of Nuclear Medicine and Molecular Imaging, Inc.

DOI: $10.2967 /$ jnumed.115.159533 opportunities, both for the study of fundamental immune processes in health and in disease and for the monitoring of responses to pharmacologic intervention.

In this issue of The Journal of Nuclear Medicine, Anna Wu et al. describe the successful imaging of secondary lymphoid organs, rich in $\mathrm{T}$ cells, using radiolabeled monoclonal antibodies, directed against the CD4 or CD8 T cell antigens (13). Specifically, the authors used antibodies in diabody format, which is cleared more rapidly than the corresponding parental IgG format and is thus more suitable for in vivo imaging applications $(14,15)$. The radiolabeled antibody fragments, which were specific to mouse CD4 and CD8 antigens, nicely imaged the lymph nodes and spleen in immunocompetent mice, with excellent selectivity (as confirmed by the quantitative assessment of percentage injected dose per gram of tissues in various organs and body structures). Several control experiments, including the administration of radiolabeled antibodies after a suitable lymphocyte depletion step with unlabeled antibodies, were performed to confirm the specificity of the imaging procedure.

The article is important for several reasons. First, the authors nicely show that the CD4- and CD8-specific diabodies are not trapped in blood by circulating lymphocytes, which are present at a lower density, compared with solid structures in secondary lymphoid organs. Second, the experiments suggest that it may be possible to gain information about $\mathrm{T}$ cell density in certain tissues (e.g., in tumors) from the corresponding signals in nuclear medicine investigations.

What can we expect from future development in the field? On one hand, it will be important to experimentally validate whether $\mathrm{T}$ cell infiltrates in tumors can be quantitatively assessed in rodent models of cancer and in patients, using suitable diabody-based imaging agents. Furthermore, similar imaging strategies could potentially be used for the in vivo monitoring of engineered $\mathrm{T}$ cell specificities (e.g., those clinically used in chimeric antibody receptor technology (10)). More broadly, the availability of surface markers for defined subsets of $\mathrm{T}$ cells (or other leukocytes) may allow a finer quantification of cellular infiltrates in organs and diseased tissue. Molecular imaging techniques may therefore become extremely important for the industrial and clinical development of immunomodulatory agents.

What will be the main future challenges? First, distinctive surface markers for many important $\mathrm{T}$ cell subtypes (e.g., regulatory $\mathrm{T}$ cells) are not yet available. Second, signal to noise in imaging procedures may decrease, if antibodies that target rare lymphocyte populations are used. On the other hand, the extensive knowledge available for the expression patterns of cluster of differentiation antigens on leukocytes in health and disease would deserve imaging investigations, with affinity reagents similar to the ones described in the current study (13). In this respect, CD4 and CD8 may represent only the beginning of a more extensive 
series of nuclear medicine investigations. Finding a balance between the regulatory need to comply with good manufacture practice guidelines and the need to clinically investigate various types of antibody molecules will also represent a formidable challenge in translational medicine.

\section{REFERENCES}

1. Alexandrov LB, Nik-Zainal S, Wedge DC, et al. Signatures of mutational processes in human cancer. Nature. 2013;500:415-421.

2. Wolchok JD, Kluger H, Callahan MK, et al. Nivolumab plus ipilimumab in advanced melanoma. N Engl J Med. 2013;369:122-133.

3. Robert C, Long GV, Brady B, et al. Nivolumab in previously untreated melanoma without BRAF mutation. N Engl J Med. 2015;372:320-330.

4. Robert C, Schachter J, Long GV, et al.; KEYNOTE-006 investigators. Pembrolizumab versus ipilimumab in advanced melanoma. $N$ Engl $\mathrm{J}$ Med. April 19, 2015 [Epub ahead of print].

5. Pasche N, Neri D. Immunocytokines: a novel class of potent armed antibodies. Drug Discov Today. 2012;17:583-590.

6. Danielli R, Patuzzo R, Di Giacomo AM, et al. Intralesional administration of L19IL2/L19TNF in stage III or stage IVM1a melanoma patients: results of a phase II study. Cancer Immunol Immunother. May 14, 2015 [Epub ahead of print].
7. Navid F, Sondel PM, Barfield R, et al. Phase I trial of a novel anti-GD2 monoclonal antibody, Hu14.18K322A, designed to decrease toxicity in children with refractory or recurrent neuroblastoma. J Clin Oncol. 2014;32: 1445-1452.

8. Schwager K, Kaspar M, Bootz F, et al. Preclinical characterization of DEKAVIL (F8-IL10), a novel clinical-stage immunocytokine which inhibits the progression of collagen-induced arthritis. Arthritis Res Ther. 2009;11:R142.

9. Hemmerle T, Doll F, Neri D. Antibody-based delivery of IL4 to the neovasculature cures mice with arthritis. Proc Natl Acad Sci USA. 2014;111:12008-12012.

10. June $\mathrm{CH}$, Riddell SR, Schumacher TN. Adoptive cellular therapy: a race to the finish line. Sci Transl Med. 2015;7:280ps7.

11. Schumacher TN, Schreiber RD. Neoantigens in cancer immunotherapy. Science. 2015;348:69-74.

12. Kvistborg P, Philips D, Kelderman S, et al. Anti-CTLA-4 therapy broadens the melanoma-reactive CD8+ T cell response. Sci Transl Med. 2014;6:254ra128.

13. Tavaré R, McCracken MN, Zettlitz KA, et al. Immuno-PET of murine T cell reconstitution postadoptive stem cell transplantation using anti-CD4 and antiCD8 Cys-diabodies. J Nucl Med. 2015;56:1258-1264.

14. Wu AM, Senter PD. Arming antibodies: prospects and challenges for immunoconjugates. Nat Biotechnol. 2005;23:1137-1146.

15. Borsi L, Balza E, Bestagno M, et al. Selective targeting of tumoral vasculature: comparison of different formats of an antibody (L19) to the ED-B domain of fibronectin. Int J Cancer. 2002;102:75-85. 